\title{
Intramedullary spinal cord metastasis from malignant melanoma: a case report of a central nervous system secondary lesion occurred 15 years after the primary skin lesion resection
}

\author{
Maciej Śniegocki ${ }^{1}$, Agnieszka Nowacka ${ }^{1}$, Wojciech Smuczyński², Kamila Woźniak ${ }^{1}$
}

1Department of Neurosurgery, Neurotraumatology and Pediatric Neurosurgery, Collegium Medicum in Bydgoszcz, Nicolaus Copernicus University in Torun, Poland

${ }^{2}$ Department of Neurotraumatology, Collegium Medicum in Bydgoszcz, Nicolaus Copernicus University in Torun, Poland

Adv Dermatol Allergol 2018; XXXV (3): 325-326

DOI: https://doi.org/10.5114/ada.2018.72760

Melanomas are malignant tumors prone to early metastasis $[1,2]$. According to the latest studies, nearly half of patients with melanoma will experience metastases to the central nervous system (CNS) [3]. Intramedullary metastases give symptoms associated with tumor mass compression on the spinal cord at a given level. The main goal of treating this type of cancer is to improve the patient's neurological state [4]. In this paper we report a case of intramedullary spinal cord metastasis from malignant melanoma detected 15 years after the primary skin lesion resection.

A 49-year-old female patient was admitted to the Department of Neurosurgery, Neurotraumatology and Pediatric Neurosurgery in March 2009 for diagnosis and treatment of back pain. The patient had previously been diagnosed with malignant melanoma (I B) of the left forearm. The lesion underwent extensive surgical resection in February 1994. No recurrence or metastasis has appeared since then. In examination on admission, the following was established: good general condition, back pain at the lower part of the thoracic spine, spasticity of the lower limbs, and no sensory disturbance. Thoracic spine magnetic resonance imaging (MRI) with contrast showed a longitudinal pathological mass $(22.8 \mathrm{~mm}$ $\times 9.8 \mathrm{~mm} \times 8.3 \mathrm{~mm}$ ) within the spinal cord at Th11 level, widening the spinal cord and following a gadoliniumcontrast injection homogeneously enhancing the surface of the spinal cord. Based on clinical symptoms and MRI image, the patient was qualified for an emergency surgical treatment.

The surgery confirmed the presence of intramedullary tumor at level Th11. On opening the dura, there ap- peared a visibly swollen area of the spinal cord at the surgery level. A linear midline incision was made, revealing tumor masses. Macroscopically the lesion was totally removed. After surgery, the patient's neurological condition improved. Early postoperative rehabilitation was implemented. Until the end of stay at the Department of Neurosurgery, gradual regression of both lower limbs paresis was observed.

In a histopathological examination, immunohistochemical staining showed positivity for HMB45 and Melan-A (Figure 1), which confirmed the diagnosis of melanoma metastasis. On the $14^{\text {th }}$ day after surgery the patient was discharged from the clinic in a good general condition, walking.

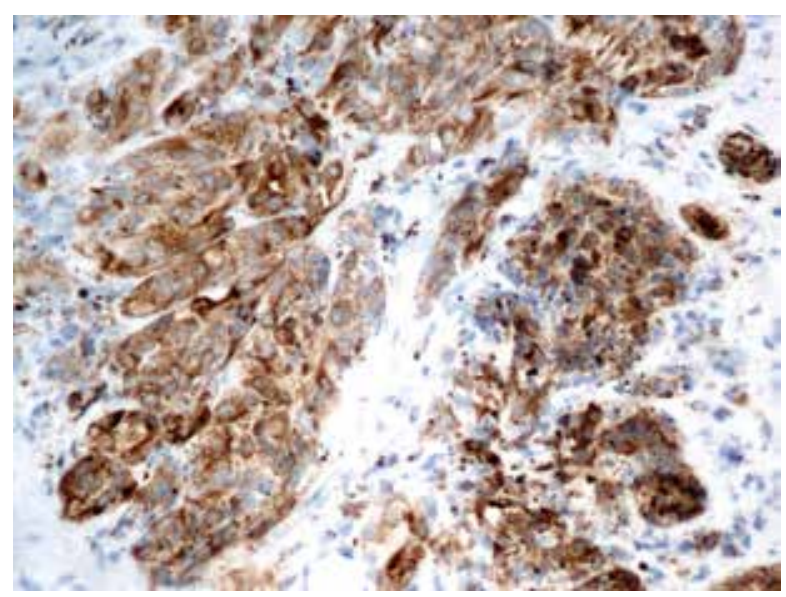

Figure 1. Immunohistochemical staining - positivity for Melan-A (20x)

Address for correspondence: Agnieszka Nowacka PhD, Department of Neurosurgery, Neurotraumatology and Pediatric Neurosurgery, Collegium Medicum, Nicolaus Copernicus University, 9 Skłodowskiej-Curie St, 85-094 Bydgoszcz, Poland, phone: +48 601644 756, e-mail: dr.agnieszka.nowacka@gmail.com Received: 11.09.2017, accepted: 12.10.2017. 
Intramedullary malignant melanoma is extremely rare [5-7]. In case of skin melanoma metastasis to the spinal cord, prognosis is worse than for primary CNS melanoma [8]. These tumors develop very fast and if untreated cause death within on average 6 months to 12 months [9-11]. In this case, metastatic melanoma was detected 15 years after the primary skin lesion resection. Malignant melanomas metastasize quickly, which is why until histopathological diagnosis, this tumor was not considered in the initial diagnosis. In the case described by Sun et al. [12], melanoma metastases were diagnosed 13 years after the primary lesion resection. This is the longest period of time from the diagnosis of primary cancer to the occurrence of secondary lesions found in the literature. However, that case was not an intramedullary lesion but a L3-L4 spinal canal extradural tumor.

Beyond the unusual time of secondary lesion occurrence in the described patient, the location of the primary lesion on the left forearm was also not indicative of melanoma. In reference to the literature, in the vast majority of spinal metastases cases, the primary lesion is located within the trunk [13].

Melanoma is highly resistant to radiation [14], therefore, despite the high risk of spinal cord damage, the CNS metastases treatment of choice is a surgical resection. Its goal is to improve patient's neurological state and functional condition, reduce the pain and thus improve the quality of life. In the presented case, surgical treatment resulted in significant improvement of neurological and functional status of the patient.

Confirmation of melanoma metastasis diagnosis is based on histopathological examination of surgically removed tumor mass. In immunohistochemical examination, melanoma is immunoreactive for HMB-45, S-100 and Melan-A [15]. In the presented case, in addition to standard histopathological examination, performance of immunohistochemical analysis with the use of recommended HMB-45 and Melan-A antibodies, allowed to make the final diagnosis of malignant melanoma metastasis.

Our report is unique in that, to the best of our knowledge, it is the first case of a CNS secondary lesion that occurred 15 years after the primary skin lesion resection. In addition, the location of the primary tumor, i.e. the left forearm, has been non-standard as for melanoma metastasis.

\section{Conflict of interest}

The authors declare no conflict of interest.

\section{References}

1. O’Day SJ, Kim CJ, Reintgen DS. Metastatic melanoma: chemotherapy to biochemotherapy. Cancer Control 2002; 9: 31-8.

2. Czarnecka A, Czarnecki R, Witkiewicz W, HryncewiczGwóźdź A. Importance of sonography of the skin and subcu- taneous tissue in the early diagnosis of melanoma in-transit metastasis with the presentation of two cases. Adv Dermatol Allergol 2018; 35: 204-7.

3. Gretz S, Poon M, Bedard G, et al. Intramedullary spinal cord metastasis from melanoma: a case report. J Pain Manage 2013; 6: 245-8.

4. Payer S, Mende KC, Westphal M, Eicker SO. Intramedullary spinal cord metastases: an increasingly common diagnosis. Neurosurg Focus 2015; 39: E15.

5. Ishii T, Terao T, Komine K, Abe T. Intramedullary spinal cord metastases of malignant melanoma: an autopsy case report and review of the literature. Clin Neuropathol 2010; 29: 334-40.

6. Amer MH, Al-Sarraf M, Baker LH, Vaitkevicius VK. Malignant melanoma and central nervous system metastases. Incidence, diagnosis, treatment and survival. Cancer 1978; 42: 660-8.

7. Connolly ES Jr, Winfree CJ, McCormick PC, et al. Intramedullary spinal cord metastasis: report of three cases and review of the literature. Surg Neurol 1996; 46: 329-37.

8. Larson TC $3^{\text {rd }}$, Houser OW, Onofrio BM, et al. Primary spinal melanoma. J Neurosurg 1987; 66: 47-9.

9. Braud F, Khayat D, Kroon B, et al. Malignant melanoma. Crit Rev Oncol Hematol 2003; 47: 35-63.

10. Traul DE, Shaffrey ME, Schiff D. Part I: Spinal cord neoplasms - intradural neoplasms. Lancet Oncol 2007; 8: 35-45.

11. Fuld AD, Speck ME, Harris BT, et al. Primary melanoma of the spinal cord: a case report, molecular footprint, and review of the literature. J Clin Oncol 2011; 29: e499-502.

12. Sun L, Song Y, Gong Q. Easily misdiagnosed delayed metastatic intraspinal extradural melanoma of the lumbar spine: a case report and review of the literature. Oncol Lett 2013; 5: 1799-802.

13. Gokaslan ZL, Aladag MA, Ellerhorst JA. Melanoma metastatic to the spine: a review of 133 cases. Melanoma Res 2000; 10: 78-80.

14. Sung WS, Sung MJ, Chan JH, et al. Intramedullary spinal cord metastases: a 20-year institutional experience with comprehensive literature review. World Neurosurg 2012; 79: 576-84.

15. Biernat W. Metastatic tumours of the central nervous system - a pathological approach. Folia Neuropathol 2009; 47: 228-33. 